\title{
Bigram statistics for 205 five-letter words having single-solution anagrams
}

\author{
K. J. GILHOOLY \\ University of Aberdeen, Aberdeen AB9 2UB, Scotland
}

\begin{abstract}
To assist research in anagram solving, this paper presents bigram statistics for 205 five-letter words known to form single-solution anagrams. Imagery, concreteness, age-of-acquisition, familiarity, and meaningfulness values for these words have previously been published. The bigram statistics presented here include the bigram rank and GTZERO measures recently devised and tested by Mendelsohn (1976).
\end{abstract}

A considerable number of solution word attributes have been implicated in anagram solving. Research in this area needs large collections of words measured on relevant attributes so that suitable word samples can be drawn. Gilhooly and Hay (1977) presented imagery, concreteness, age-of-acquisition, familiarity, and meaningfulness values for 205 five-letter words taken from Olson and Schwartz's (1967) list of words having singlesolution anagrams. The Gilhooly and Hay list did not include bigram measures, but some recent research (Mendelsohn, 1976; Mendelsohn, \& O'Brien, 1974) has uncovered two influential measures based on bigram frequencies that should be taken into account in using such lists. This paper reports the relevant bigram measures for the words in the Gilhooly and Hay list.

The first of these new measures is the total bigram rank, allowing for bigram position and word length. Mendelsohn (1976) labeled this variable "BR." The measure is obtained using Mayzner and Tresselt's (1965) tables in the way explained by Mendelsohn and O'Brien (1974). For five-letter words, the method involves drawing up a bigram frequency matrix of 20 rows and four columns for each word. In the matrix, the rows represent the 20 possible bigrams that can be formed from five different letters and the columns represent the four bigram positions in a five-letter word. From Mayzner and Tresselt's tables the frequency of each of the 20 possible bigrams in each of the four positions is obtained. Then the number of entries in the matrix that have higher frequencies than the four correct entries is obtained. The resulting number is the BR score for the word. This measure was found to be highly predictive of anagram difficulty (Mendelsohn, 1976; Mendelsohn \& O'Brien, 1974), in that low BR facilitated solution. Presumably, this was because the greater the relative frequency of occurrence of correct bigrams, within the set of bigrams that can be made from a given

Requests for reprints should be sent to K. J. Gilhooly, Department of Psychology, University of Aberdeen, Aberdeen AB9 2UB, Scotland. word, the more likely the subject is to generate a correct bigram as a memory probe.

The second measure is the number of nonzero entries in the bigram frequency matrix. Mendelsohn (1976) found a separate negative effect on anagram solving for this variable (which he labeled "GTZERO").

The negative correlation between GTZERO and solution rate probably arises because the greater the number of nonzero entries, the fewer the bigram possibilities that can be ruled out and the larger the set of plausible bigrams to be searched for the correct ones.

Gilhooly and Johnson (1978) confirmed the importance of BR and GTZERO in predicting anagram difficulty and also found that rated age of acquisition and familiarity were correlated significantly with BR. Apparent age effects on anagram solving dwindled when BR was partialled out. Given the importance of BR and GTZERO, and their possible confounding with other variables, it follows that these measures should be taken into account in future anagram studies. However, as will be apparent from the foregoing description, the procedure for obtaining the BR and GTZERO scores by hand is tedious, time consuming, and prone to error. To overcome this problem a FORTRAN program (PROG.BR) was written that does all the necessary table searching and calculations for five-letter strings. The program was applied to the 205 words of the Gilhooly and Hay (1977) list to produce the bigram statistics listed in the Appendix. These additional statistics should add to the usefulness of the Gilhooly and Hay list, especially for anagram studies.

The program incorporates Mayzner and Tresselt's (1965) frequency data for all bigrams in Bigram Positions 14 of five-letter words. When a five-letter string is input, the program draws up the complete 20 by 4 bigram frequency matrix for that string. Optionally, the program will output any or all of the following, (1) the complete 20 by 4 matrix, (2) the correct bigram frequencies, (3) the total correct bigram frequency, (4) the BR score, and (5) the GTZERO score. The output of the program was checked against a sample of 80 handscored words and proved completely accurate. 
Table 1

Bigram Measures: Correlations and Summary Statistics

\begin{tabular}{clccccccc}
\hline Bigram & & & & & & \\
Measure & & 1 & 2 & 3 & 4 & 5 & \multicolumn{1}{c}{7} \\
\hline 1 & BFOT & 1.00 & $.59^{*}$ & $.64^{*}$ & $.47^{*}$ & $.57^{*}$ & $-.60^{*}$ & .01 \\
2 & BF1 & & 1.00 &. $.39^{*}$ & .09 & .01 & $-.35^{*}$ & -.09 \\
3 & BF2 & & & 1.00 & .07 & -.02 & $-.42^{*}$ & -.05 \\
4 & BF3 & & & & 1.00 & .07 & $-.39^{*}$ & .05 \\
5 & BF4 & & & & & 1.00 & $-.25^{*}$ & .09 \\
6 & BR & & & & & & 1.00 & $.44^{*}$ \\
7 & GTZERO & & & & & & & 1.00 \\
& Mean & 148.86 & 31.71 & 41.67 & 30.64 & 44.83 & 52.04 & 37.90 \\
& SD & 108.96 & 39.90 & 52.30 & 38.99 & 59.43 & 31.87 & 7.97 \\
\hline
\end{tabular}

*Indicates correlations significant at the .05 level, on a two-tailed test with $d f=203$.

Table 2

Correlations Between Bigram Measures and Word Attributes

\begin{tabular}{lccccccr}
\hline & BFTOT & BF1 & BF2 & BF3 & BF4 & BR & GTZERO \\
\hline Imagery & $.15^{*}$ & $.14^{*}$ & .11 & -.00 & .08 & -.09 & -.05 \\
Concreteness & $.14^{*}$ & .12 & .07 & -.01 & .12 & -.05 & -.04 \\
Age of Acquisition & $-.32^{*}$ & $-.20^{*}$ & $-.25^{*}$ & -.09 & $-.18^{*}$ & $.35^{*}$ & .13 \\
Familiarity & $.33^{*}$ & $.19^{*}$ & $.27^{*}$ & $.16^{*}$ & .14 & $-.38^{*}$ & -.10 \\
Meaningfulness & $.26^{*}$ & $.17^{*}$ & $.15^{*}$ & .11 & $.16^{*}$ & $-.22^{*}$ & -.03 \\
Thorndike-Lorge Frequency & $.43^{*}$ & $.19^{*}$ & $.36^{*}$ & $.23^{*}$ & $.19^{*}$ & $-.39^{*}$ & .02 \\
\hline
\end{tabular}

*Indicates correlations significant at the .05 level, on a two-tailed test with $d f=203$.

The program accepts any number of five-letter strings and a listing can be obtained from the author.

\section{RESULTS AND DISCUSSION}

In addition to $B R$ and GTZERO scores, the frequencies of the four correct bigrams (BF1, BF2, BF3, $\mathrm{BF} 4)$ and the total frequency of the correct bigrams (BFTOT) are presented for each word in the Appendix. Experimenters may wish to take these into account as well as the newer measure defined by Mendelsohn (1976).

The summary statistics and intercorrelations of the bigram measures are given in Table 1. The BR-GTZERO correlation matches that obtained by Mendelsohn (1976).

In Table 2 are presented correlations between the bigram measures and the word attributes previously reported by Gilhooly and Hay (1977). The relevant correlations closely match those obtained in the subsample of 80 words examined in Gilhooly and Johnson's (1978) anagram study. Notably, the correlation of BR with age is maintained in this larger sample at about the same level as before.

\section{Appendix}

The following is an alphabetical listing of the 205 words together with each word's frequency on Bigrams $1,2,3,4$ (BF1-BF4), total correct bigram frequency (BFTOT), bigram rank (BR), and GTZERO (GT0).

\begin{tabular}{crrrrrrr}
\hline Word & BF1 & BF2 & BF3 & BF4 & BFTOT & BR & GT0 \\
\hline AGONY & 19 & 0 & 66 & 13 & 98 & 60 & 45
\end{tabular}

\begin{tabular}{lrrrrrrr}
\hline Word & BF1 & BF2 & BF3 & BF4 & BFTOT & BR & GT0 \\
\hline ALBUM & 38 & 0 & 0 & 0 & 38 & 120 & 40 \\
ANKLE & 4 & 0 & 0 & 59 & 63 & 129 & 47 \\
APRON & 6 & 0 & 5 & 1 & 12 & 149 & 51 \\
BANIO & 15 & 38 & 0 & 0 & 53 & 69 & 31 \\
BATCH & 15 & 47 & 14 & 126 & 202 & 35 & 32 \\
BATON & 15 & 47 & 2 & 1 & 65 & 90 & 46 \\
BENCH & 41 & 12 & 27 & 126 & 206 & 22 & 27 \\
BERTH & 41 & 10 & 32 & 66 & 149 & 57 & 37 \\
BIGOT & 13 & 101 & 2 & 8 & 124 & 48 & 38 \\
BIRCH & 13 & 70 & 3 & 126 & 212 & 29 & 29 \\
BITCH & 13 & 9 & 14 & 126 & 162 & 36 & 32 \\
BLACK & 18 & 56 & 51 & 37 & 162 & 15 & 30 \\
BLADE & 18 & 56 & 15 & 11 & 100 & 61 & 46 \\
BLAZE & 18 & 56 & 2 & 2 & 78 & 67 & 35 \\
BLIMP & 18 & 17 & 8 & 3 & 46 & 34 & 36 \\
BLOCK & 18 & 67 & 7 & 37 & 129 & 22 & 28 \\
BRAND & 49 & 50 & 38 & 51 & 188 & 12 & 37 \\
BRAWL & 49 & 50 & 0 & 0 & 99 & 68 & 32 \\
BRICK & 49 & 49 & 129 & 37 & 264 & 9 & 32 \\
BRINE & 49 & 49 & 142 & 27 & 267 & 35 & 48 \\
BRINK & 49 & 49 & 142 & 34 & 274 & 9 & 32 \\
BUNCH & 26 & 16 & 27 & 126 & 195 & 7 & 17 \\
BUYER & 26 & 0 & 3 & 253 & 282 & 58 & 31 \\
CABIN & 29 & 6 & 1 & 32 & 68 & 62 & 41 \\
CABLE & 29 & 6 & 5 & 59 & 99 & 73 & 44 \\
CAIRN & 29 & 21 & 12 & 6 & 68 & 82 & $\mathbf{5 4}$ \\
CHAIR & 40 & 33 & 40 & 73 & 186 & 35 & 44 \\
CHALK & 40 & 33 & 27 & 0 & 100 & 49 & 29 \\
CHILD & 40 & 194 & 90 & 160 & 484 & 5 & 29 \\
CHIME & 40 & 194 & 8 & 5 & 247 & 47 & 41 \\
CHIMP & 40 & 192 & 8 & 3 & 245 & 21 & 31 \\
CHORD & 40 & 78 & 36 & 23 & 177 & 21 & 34 \\
CHUMP & 40 & 4 & 3 & 3 & 50 & 32 & 22 \\
CHUNK & 40 & 4 & 59 & 34 & 137 & 15 & 21 \\
CLAMP & 43 & 56 & 5 & 3 & 107 & 46 & 36 \\
CLASH & 43 & 56 & 48 & 13 & 160 & 24 & 36 \\
CLERK & 43 & 12 & 152 & 1 & 208 & 54 & 39 \\
CLOAK & 43 & 67 & 4 & 20 & 134 & 42 & 41 \\
& & & & & & &
\end{tabular}




\begin{tabular}{|c|c|c|c|c|c|c|c|c|c|c|c|c|c|c|c|}
\hline Word & BF1 & BF2 & BF3 & BF4 & BFTOT & BR & GT0 & Word & BF1 & BF2 & BF3 & BF4 & BFTOT & BR & GT0 \\
\hline CLOTH & 43 & 67 & 11 & 66 & 187 & 35 & 38 & IDLER & 8 & 0 & 43 & 253 & 304 & 107 & 57 \\
\hline CLOWN & 43 & 67 & 23 & 12 & 145 & 18 & 30 & INDEX & 1 & 21 & 41 & 0 & 63 & 92 & 42 \\
\hline CORGI & 72 & 85 & 20 & 0 & 177 & 64 & 48 & INPUT & 1 & 0 & 0 & 92 & 93 & 120 & 44 \\
\hline COUNT & 72 & 222 & 59 & 44 & 397 & 9 & 46 & JUDGE & 3 & 6 & 7 & 33 & 49 & 44 & 26 \\
\hline COUGH & 72 & 222 & 8 & 6 & 308 & 26 & 31 & JUICE & 3 & 34 & 129 & 76 & 242 & 18 & 27 \\
\hline COVER & 72 & 16 & 74 & 253 & 415 & 24 & 48 & JOINT & 2 & 65 & 142 & 44 & 253 & 29 & 40 \\
\hline CRAFT & 20 & 50 & 0 & 3 & 73 & 81 & 41 & KNAVE & 13 & 1 & 19 & 45 & 78 & 77 & 40 \\
\hline CRAMP & 20 & 50 & 5 & 3 & 78 & 60 & 39 & KNIFE & 13 & 2 & 2 & 0 & 17 & 112 & 39 \\
\hline CRANK & 20 & 50 & 38 & 34 & 142 & 23 & 42 & LARCH & 36 & 51 & 3 & 126 & 216 & 34 & 36 \\
\hline CRAZE & 20 & 50 & 2 & 2 & 74 & 82 & 40 & LIGHT & 36 & 101 & 94 & 95 & 326 & 11 & 33 \\
\hline CROFT & 20 & 64 & 1 & 3 & 88 & 77 & 43 & LIMBO & 36 & 13 & 1 & 0 & 50 & 96 & 45 \\
\hline CROWD & 20 & 64 & 23 & 3 & 110 & 40 & 35 & LOGIC & 23 & 2 & 7 & 18 & so & 65 & 40 \\
\hline CRUMB & 20 & 14 & 3 & 5 & 42 & 28 & 26 & LOVER & 23 & 16 & 74 & 253 & 366 & 40 & 48 \\
\hline CRUSH & 20 & 14 & 22 & 13 & 69 & 26 & 33 & LUNCH & 4 & 16 & 27 & 126 & 173 & 23 & 22 \\
\hline CRYPT & 20 & 0 & 0 & 4 & 24 & 57 & 23 & LYRIC & 4 & 0 & 2 & 18 & 24 & 85 & 33 \\
\hline DEMON & 15 & 2 & 1 & 1 & 19 & 128 & 49 & MAJOR & 38 & 6 & 10 & 27 & 81 & 51 & 39 \\
\hline DEPTH & 15 & 4 & 6 & 66 & 91 & 61 & 38 & MAKER & 38 & 33 & 57 & 253 & 381 & 26 & 48 \\
\hline DIVOT & 9 & 36 & 1 & 8 & 54 & 76 & 42 & MATCH & 38 & 47 & 14 & 126 & 225 & 27 & 35 \\
\hline DOUBT & 18 & 222 & 7 & 2 & 249 & 47 & 37 & MEDAL & 16 & 1 & 10 & 35 & 62 & 104 & 52 \\
\hline DRAWL & 25 & 50 & 0 & 0 & 75 & 87 & 38 & MERCY & 16 & 10 & 3 & 0 & 29 & 79 & 34 \\
\hline DRINK & 25 & 49 & 142 & 34 & 250 & 14 & 39 & MIDGE & 36 & 9 & 7 & 33 & 85 & 52 & \\
\hline DRUNK & 25 & 14 & 59 & 34 & 132 & 8 & 33 & MINCE & 36 & 27 & 27 & 76 & 166 & 24 & 4 \\
\hline DUNCE & 6 & 16 & 27 & 76 & 125 & 44 & 41 & MINER & 36 & 27 & 24 & 253 & 340 & 44 & \\
\hline ENTRY & 14 & 9 & 1 & 50 & 74 & 91 & 42 & MIRTH & 36 & 70 & 32 & 66 & 204 & 26 & \\
\hline FAIRY & 15 & 21 & 12 & 50 & 98 & 41 & 36 & MIXER & 36 & 3 & 7 & 253 & 299 & 60 & 4 \\
\hline FAITH & 15 & 21 & 31 & 66 & 133 & 44 & 38 & MONTH & 48 & 32 & 22 & 66 & 168 & 31 & 3 \\
\hline FANCY & 15 & 38 & 27 & 0 & 80 & 36 & 27 & MOUTH & 48 & 222 & 22 & 66 & 358 & 25 & 3 \\
\hline FAULT & 15 & 7 & 131 & 15 & 168 & 57 & 44 & NYMPH & 1 & 1 & 4 & 2 & 8 & 28 & 14 \\
\hline FIGHT & 63 & 101 & 94 & 95 & 353 & 9 & 28 & ODIUM & 3 & 0 & 0 & 0 & 3 & 154 & 43 \\
\hline FILTH & 63 & 27 & 3 & 66 & 159 & 38 & 33 & OPERA & 2 & 26 & 152 & 2 & 182 & 98 & \\
\hline FINCH & 63 & 27 & 27 & 126 & 243 & 16 & 23 & OPIUM & 2 & 3 & 0 & 0 & 5 & 141 & 4 \\
\hline FLAKE & 22 & 56 & 3 & 13 & 94 & 58 & 37 & ORBIT & 10 & 3 & 1 & 10 & 24 & 124 & 5 \\
\hline FLAME & 22 & 56 & 5 & 5 & 88 & 73 & 44 & OUNCE & 6 & 16 & 27 & 76 & 125 & 46 & 4 \\
\hline FLANK & 22 & 56 & 38 & 34 & 150 & 16 & 29 & OVARY & 0 & 0 & 102 & 50 & 152 & 79 & \\
\hline FLASH & 22 & 56 & 48 & 13 & 139 & 24 & 30 & PANIC & 30 & 38 & 1 & 18 & 87 & 55 & \\
\hline FLASK & 2 & 56 & 48 & 0 & 126 & 38 & 32 & PER & 2 & 10 & 3 & 126 & 141 & 71 & \\
\hline FLIRT & 22 & 17 & 12 & 26 & 77 & 59 & 41 & PILC & 10 & 27 & 9 & 8 & 54 & 74 & 5 \\
\hline FLUKE & 22 & 20 & 0 & 13 & 5 & 45 & 30 & & 10 & 9 & 14 & 126 & 159 & 37 & \\
\hline FORCE & 31 & 85 & 3 & 76 & 195 & 43 & 46 & PIVO & 10 & 36 & 1 & 8 & 55 & 68 & \\
\hline FORUM & 31 & 85 & 2 & 0 & 11 & 71 & 39 & PLANK & 27 & 56 & 38 & 34 & 155 & 14 & \\
\hline FOVEA & 31 & 16 & 74 & 0 & 121 & 52 & 36 & PLANT & 27 & 56 & 38 & 44 & 165 & 13 & \\
\hline FRAUD & 16 & 50 & 0 & 7 & 73 & 67 & 42 & PLU & 27 & 20 & 12 & 37 & 96 & 10 & \\
\hline FRONT & 16 & 64 & 66 & 44 & 190 & 21 & 42 & PLUI & 27 & 20 & 3 & 5 & 55 & 57 & \\
\hline FROWN & 16 & 64 & 23 & 12 & 115 & 29 & 30 & POR & 31 & 85 & 3 & 126 & 245 & 28 & \\
\hline FRUIT & 16 & 14 & 4 & 10 & 44 & 75 & 46 & & 19 & 5 & 38 & 34 & 14 & 17 & 3 \\
\hline GAUZE & 7 & 7 & 0 & 2 & 16 & 76 & 29 & & 19 & 5 & 0 & 12 & 8 & 50 & 3 \\
\hline GLAND & 15 & 56 & 38 & 51 & 16 & 20 & 41 & & 19 & 49 & & 2 & 72 & 74 & \\
\hline GLEAM & 15 & 12 & 7 & 6 & 10 & 72 & 46 & & 19 & 14 & 59 & 27 & 119 & 37 & 4 \\
\hline GLINT & 15 & 1 & 142 & 44 & & 27 & & & 0 & 0 & 9 & 1 & 10 & 104 & \\
\hline GLOI & 15 & 67 & 36 & 50 & 16 & 19 & 34 & QUERY & 27 & 10 & 15 & 50 & 23 & 20 & \\
\hline & 15 & 67 & 17 & 45 & 144 & 27 & 39 & & 27 & 34 & 9 & 15 & 16 & 20 & \\
\hline $\mathrm{GN}$ & 0 & 9 & 1 & 5 & 15 & 119 & 45 & & 23 & 38 & 27 & 126 & 214 & 26 & 3 \\
\hline & 41 & 50 & 0 & 3 & 9 & 70 & 39 & & 0 & 0 & 0 & 5 & 5 & 105 & \\
\hline & 41 & 50 & 38 & 44 & 17 & 19 & 49 & & 50 & 36 & 74 & 18 & 178 & 48 & \\
\hline GRAPH & 41 & 50 & 8 & 2 & 10 & 49 & 37 & & 23 & 9 & 10 & 253 & 29 & 76 & \\
\hline & 41 & 50 & 19 & 45 & & 46 & 4 & & 13 & 4 & 0 & 2 & 1 & 58 & \\
\hline GRIEF & 41 & 49 & 30 & 6 & 12 & 59 & 43 & & 7 & 0 & 102 & 1 & 110 & 73 & \\
\hline & 41 & 64 & 107 & 6 & & 23 & 46 & & 7 & 2 & 107 & 92 & 208 & 58 & \\
\hline & 14 & 3 & 102 & 23 & 14 & 51 & 51 & SCRI & 7 & 1 & 2 & 0 & 10 & 94 & \\
\hline GUILT & 14 & 34 & 90 & 15 & 15 & 32 & 41 & & 7 & 0 & 0 & 66 & 73 & 64 & \\
\hline HAVEN & 22 & 9 & 74 & 75 & 18 & 43 & 36 & SH & 62 & 33 & 0 & 0 & 95 & 69 & \\
\hline HAVOC & 22 & 9 & 1 & 0 & 32 & 77 & 32 & & 0 & 1 & 51 & 37 & 89 & 70 & \\
\hline HONEY & 59 & 32 & 24 & 20 & 135 & 30 & 35 & SQUIB & 1 & 2 & 4 & 0 & 7 & 77 & \\
\hline & 59 & 85 & 12 & 11 & 16 & 57 & 44 & STYLE & 136 & 7 & 7 & 59 & 209 & 57 & \\
\hline HOUND & 59 & 222 & 59 & 51 & 39 & 9 & 33 & TEMPO & 20 & 2 & 4 & 1 & 27 & 110 & \\
\hline & 59 & 222 & 22 & 149 & 452 & 19 & 37 & THIEF & 302 & 194 & 30 & 6 & 532 & 38 & \\
\hline & 59 & 16 & 74 & 10 & 159 & 38 & 36 & THUMB & 302 & 4 & 3 & 5 & 314 & 33 & \\
\hline HYENA & 0 & 0 & 20 & 5 & 25 & 111 & 37 & TOKEN & 27 & 8 & 57 & 75 & 167 & 37 & \\
\hline
\end{tabular}




\begin{tabular}{lrrrrrrr}
\hline Word & BF1 & BF2 & BF3 & BF4 & BFTOT & BR & GT0 \\
\hline TRAMP & 45 & 50 & 5 & 3 & 103 & 64 & 44 \\
TREND & 45 & 61 & 20 & 51 & 177 & 39 & 48 \\
TRICK & 45 & 49 & 129 & 37 & 260 & 9 & 42 \\
TRUCK & 45 & 14 & 12 & 37 & 108 & 16 & 34 \\
TRUMP & 45 & 14 & 3 & 3 & 65 & 48 & 36 \\
TULIP & 2 & 23 & 6 & 1 & 32 & 98 & 47 \\
UNCLE & 25 & 0 & 0 & 59 & 84 & 91 & 41 \\
UNITY & 25 & 2 & 31 & 23 & 81 & 47 & 38 \\
VALET & 4 & 31 & 43 & 18 & 96 & 77 & 47 \\
VAULT & 4 & 7 & 131 & 15 & 157 & 56 & 38 \\
VENOM & 1 & 12 & 1 & 2 & 16 & 114 & 43 \\
VICAR & 8 & 5 & 3 & 13 & 29 & 93 & 44 \\
VIPER & 8 & 1 & 19 & 253 & 281 & 84 & 47 \\
VIRUS & 8 & 70 & 2 & 0 & 80 & 84 & 36 \\
VISOR & 8 & 10 & 0 & 27 & 45 & 97 & 42 \\
VISTA & 8 & 10 & 16 & 0 & 34 & 109 & 43 \\
VIXEN & 8 & 3 & 7 & 75 & 93 & 72 & 36 \\
VODKA & 13 & 11 & 0 & 0 & 24 & 78 & 31 \\
WALTZ & 42 & 31 & 3 & 0 & 76 & 47 & 27 \\
WATCH & 42 & 47 & 14 & 126 & 229 & 28 & 33 \\
WENCH & 16 & 12 & 27 & 126 & 181 & 32 & 32 \\
WHARF & 187 & 33 & 102 & 1 & 323 & 22 & 23 \\
WHEAT & 187 & 263 & 75 & 7 & 542 & 24 & 41 \\
WIDTH & 9 & 9 & 1 & 66 & 85 & 48 & 28 \\
WITCH & 9 & 9 & 14 & 126 & 158 & 43 & 31 \\
WOMAN & 163 & 45 & 23 & 40 & 271 & 12 & 42 \\
WORLD & 163 & 85 & 44 & 160 & 452 & 2 & 36 \\
WOUND & 163 & 222 & 59 & 51 & 495 & 4 & 38 \\
WRECK & 16 & 61 & 8 & 37 & 122 & 30 & 34 \\
YOUTH & 26 & 222 & 22 & 66 & 336 & 27 & 34 \\
\hline & & & & & & &
\end{tabular}

\section{REFERENCES}

Gilhooly, K. J., \& HaY, D. Imagery, concreteness, age-ofacquisition, familiarity, and meaningfulness values for 205 five-letter words having single solution anagrams. Behavior Research Methods \& Instrumentation, 1977, 9, 12-17.

Gilhooly, K. J., \& Johnson, C. E. Effects of solution word attributes on anagram difficulty: A regression analysis. Quarterly Joumal of Experimental Psychology, 1978, 30, 57-70.

Mayzner, M. A., \& Tresselt, M. E. Tables of singleletter and bigram frequency counts for various word length and letter position combinations. Psychonomic Monograph Supplements, 1965, 1, 13-31.

Mendelsohn, G. A. An hypothesis approach to the solution of anagrams. Memory \& Cognition, 1976, 4, 637-642.

Mendelsohn, G. A., \& O'Brien, A. T. The solution anagrams: A re-examination of the effects of transition letter probabilities, letter moves, and word frequency on anagram difficulty. Memory \& Cognition, 1974, 2, 566-574.

Olson, R., \& SChwartz, R. Single- and multiple-solution five-letter words. Psychonomic Monograph Supplements, 1967, 2(8, Whole No. 24). 